\title{
Lévy flight of superdiffusive light in coupled waveguide lattices
}

\author{
Sayan Bhattacherjee, ${ }^{1}$ Sushil Mujumdar, ${ }^{2}$ and Somnath Ghosh $\odot^{1, *}$ \\ ${ }^{1}$ Unconventional Photonics Group, Department of Physics, Indian Institute of Technology Jodhpur, Rajasthan-342037, India \\ ${ }^{2}$ NOMOL, Tata Institute of Fundamental Research, Mumbai, Maharashtra 400005, India
}

(Received 27 October 2019; accepted 6 January 2020; published 12 February 2020)

\begin{abstract}
Exotic light behavior in certain disordered one-dimensional optical waveguide lattices with selectively doped gain/loss operating away from the $P T$ symmetry regime is reported. Here, we establish that such open systems host a unique Lévy flight even in the absence of scatterers following Lévy distribution. Owing to the interplay between gain/loss and disorder, the critical transition distance from the ballistic to diffusive domain is reduced and also a superdiffusive propagation distance has been revealed after a certain propagation distance. The stable propagation characteristics of light have been investigated in the form of an intensity distribution for various gain/loss levels, effective beam width variation with length, the center of mass dynamics, and the probability density function. A qualitative analysis indicates that such fabrication feasible lattices may host disordered lasing in the form of a Lévy-type distribution in the output intensity and may also find applications in image transport.
\end{abstract}

DOI: 10.1103/PhysRevResearch.2.012037

\section{INTRODUCTION}

Certain deliberate disorder causes Bloch waves to localize in otherwise periodic lattices resulting in transverse Anderson localization $[1,2]$. However, a certain localization regime exists where the logarithm of the transverse beam width is proportional to the logarithm of the propagation distance, known as anomalous localization [3]. Such a phenomenon occurs owing to an enhancement in nonuniformity in a disordered system. In this regime, due to random scattering, the photons mimic a Lévy-flight-like random walk which can be identified by the heavy-tailed nature of transverse intensity fluctuations. A special case of random walk in which extremely long jumps can occur are called Lévy flight [4]. Such light dynamics can be observed in the mesoscopic domain where scattering length scales can be tuned. In this context, evanescently coupled waveguide arrays forming a lattice are a versatile platform for studying such a quantum inspired photonic phenomenon [5]. By controlled and deliberate disorder the absorption length scale in such structures can be modified, which tunes the scattering points. At the scattering points, waves interfere, but can only be observed if the absorption lengths are larger than the distance between the scattering points. The outputs of these disordered lattices are predicted through ensemble-averaged quantities. Any introduction of gain/loss makes the refractive index complex, resulting in structures that are non-Hermitian in nature. Thus understanding the many fundamental and intriguing effects and their applications in the photonics domain

\footnotetext{
*somiit@ rediffmail.com

Published by the American Physical Society under the terms of the Creative Commons Attribution 4.0 International license. Further distribution of this work must maintain attribution to the author(s) and the published article's title, journal citation, and DOI.
}

will be straightforward if we study the dynamics of wave propagation through these non-Hermitian one-dimensional (1D) lattice structures [6-11]. More interestingly, the introduction of gain has opened up a new avenue to explore the working of disordered lasers by exploiting the superdiffusive nature of light through these 1D disordered optical lattices.

Here, we report a very special kind of lattice where complex coupling has been patterned by selectively doping the bulk with an active material or customized loss. It has been established that the introduction of a little gain in the presence of adequate disorder has a significant impact on the light dynamics. Under these circumstances, the interplay of disorder and complex coupling has dictated the light will propagate in a superdiffusive regime and ultimately result in a Lévy-like distribution of the ensemble-averaged intensity profile. The Lévy distribution of light is otherwise possible if the scatterers follow the Lévy distribution pattern as in Lévy glass [4]. However, unlike previous studies where the scatterers within the material follow Lévy statistics to generate a Lévy-like intensity distribution, we have reported a Lévy-like intensity pattern out of a $1 \mathrm{D}$ waveguide lattice which is more versatile and fabrication feasible. Thus the proposed design of a 1D lattice generates a Lévy-like intensity distribution and in turn is suitable for the onset of a random lasing phenomenon, and may also find applications in image transport [12-18].

\section{MODELING OF THE LATTICE}

To establish our claim we have studied the propagation dynamics of a Gaussian beam through an evanescently coupled non-Hermitian lattice. The wavelength of the beam is chosen at $980 \mathrm{~nm}$ in such a way that the individual waveguide supports only the fundamental mode, thus forming a supermode at the end of the lattice. Owing to the non-Hermitian nature of the lattice with a low-index contrast, the scalar wave propagation dynamics is mapped onto a scalar beam 


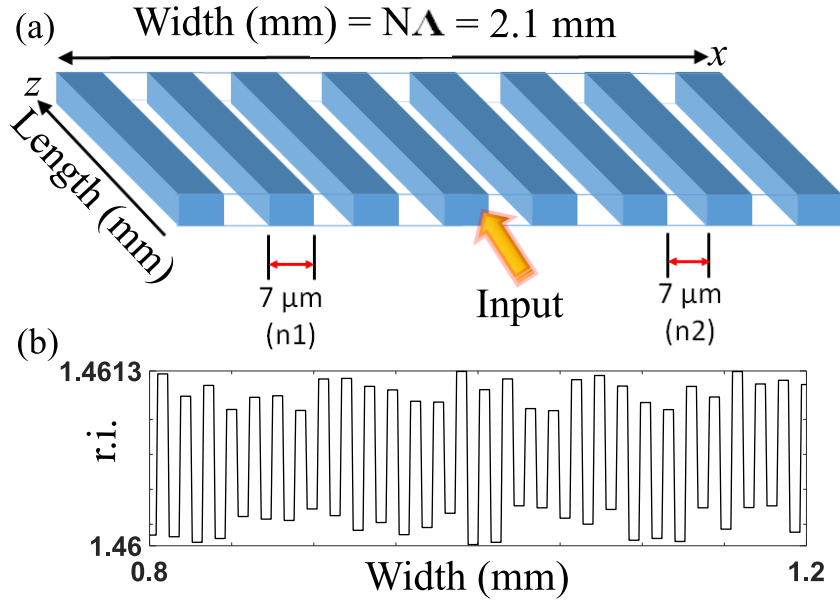

FIG. 1. Non-Hermitian 1D photonic lattice. Top: Schematic of the proposed 1D waveguide lattice through which the light beam propagates, launched at the central region denoted by the arrow. Bottom: Transverse refractive index profile indicating 30\% disorder imposed upon the periodic low- and high-index material having a value of 1.46 and 1.4613 , respectively.

propagation equation under a paraxial approximation,

$$
i \frac{\partial A}{\partial z}+\frac{1}{2 k}\left(\frac{\partial^{2} A}{\partial x^{2}}+\frac{\partial^{2} A}{\partial y^{2}}\right)+\frac{k}{n_{0}} \Delta n(x, y) A=0,
$$

where $A$ represents the amplitude of the wave, $k$ is the wave vector, and $\Delta n(x, y)$ is the variation over a constant background refractive index $n_{0}$. Now, this imposed variation over the constant background refractive index can be customized by the following equation,

$$
\Delta n(x, y)=\Delta n_{p}(x) H(x)+\Delta n_{p}(x) C \delta H(x)+i[\operatorname{Im}[\Delta n(x)]],
$$

where first and second terms denote the real part of the refractive index profile, and the third term patterns the imaginary part. The first term hosts the periodic potential, and added to it is the second part denoting the disorderness defined by a random component $\delta$ which is controlled by the dimensionless constant $C$. The schematic of the proposed lattice along with the refractive index profile is shown in Fig. 1. From Fig. 1 it can be noted that the refractive index varies between 1.46 and 1.4613 , denoting a $30 \%$ disorder over the background material of refractive index 1.46 with periodicity 0.001 . Practically, such a type of index variation can be obtained by selective doping and ultrafast laser inscription [19]. Thus to ensure $30 \%$ refractive index disorder, the $C$ value is chosen to be 0.3 and $\delta$ realizes the random distribution. However, in this Rapid Communication, we have not introduced any spatial disorder to the waveguides. The proposed lattice consists of a bulk material having a refractive index of 1.46 onto which 150 waveguides of individual width $7 \mu \mathrm{m}$ are embedded with a higher refractive index at a separation of $7 \mu \mathrm{m}$. Now, to ensure the non-Hermitian nature of the lattice, a selective gain and loss is introduced to alternate waveguides as a special case. In one of the realizations gain is introduced to the waveguide regimes whereas loss is introduced to the background material, and vice versa in the other case. The amount of gain and loss is fixed at a value 0.0001 (loss/gain coefficient) to
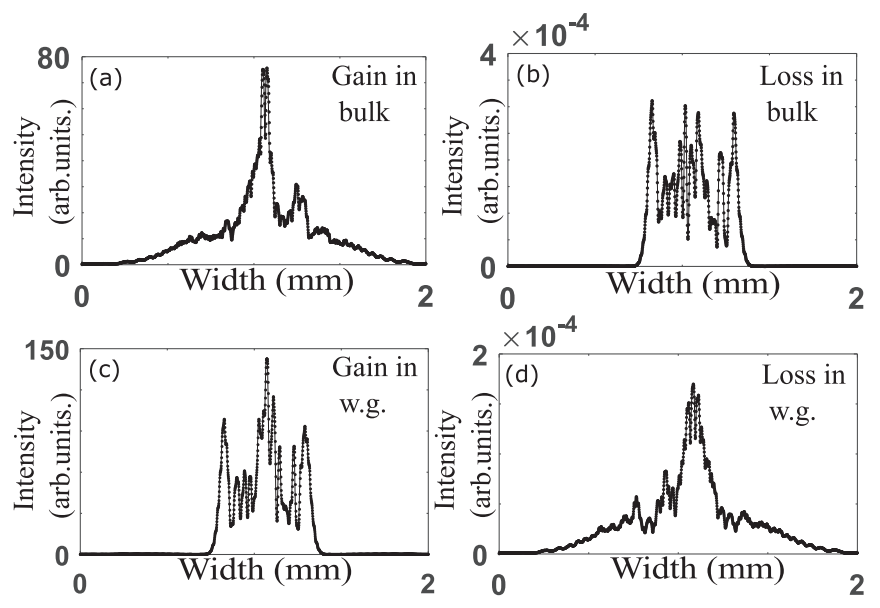

FIG. 2. Output light intensity distributions: Ensemble-averaged light intensity fluctuations at the end of a $20 \%$ disordered lattice of length $20 \mathrm{~mm}$ due to (a) application of homogeneous gain of 0.0001 to the bulk material of the lattice, (b) application of homogeneous loss of 0.0001 to the bulk material of the lattice, (c) introduction of fixed gain of 0.0001 only to high refractive index layers of the lattice, (d) and introduction of constant loss of 0.0001 only to high refractive index layers of the lattice.

avoid the onset of any gain guided mode. To maintain an equal amount of net gain and loss, the waveguides were chosen to be of equal width of $7 \mu \mathrm{m}$. The simulation of light propagation through this lattice has been performed assuming a negligible band-gap effect at the operating condition. Now, ensuring the above-mentioned criterion, we have launched a Gaussian beam with plane wave fronts having a beam width [full width at half maximum (FWHM)] of $7 \mu \mathrm{m}$ at the 76th unit cell position of the lattice. The state of light supported at the other end of the lattice due to a light-matter interaction and an interference effect is obtained by ensemble averaging the output complex scalar field through various realizations of disordered lattices. This effect of the interplay between disorder and selective gain/loss is shown in Fig. 2. Four different conditions are achieved: applying homogeneous gain $(0.0001)$ to the bulk material of the lattice, applying homogeneous loss (0.0001) to the bulk material of the lattice, introducing an equal amount of gain (0.0001) only to the high refractive index layers of the lattice, and introducing an equal amount of loss (0.0001) only to the high refractive index layers of the lattice, respectively. The ensemble-averaged output intensity distributions of 50 realizations for the four such configurations through a 20-mm lattice in the presence of $20 \%$ disorder are shown in Fig. 2. It can be observed that the introduction of gain in the background material results in complex coupling among the waveguides of the lattice. Similarly, an introduction of gain to the waveguides gives rise to its non-Hermitian nature which opens up different open channels facilitating coupling between them. From Fig. 2 it can be concluded that the introduction of homogeneous gain in the background material has a similar effect to introducing an equal amount of loss to all the waveguides. By properly assessing the behavior, it can be concluded that light propagation enters the diffusive domain from a ballistic domain due to the introduction of homogeneous gain in the background material or introducing 

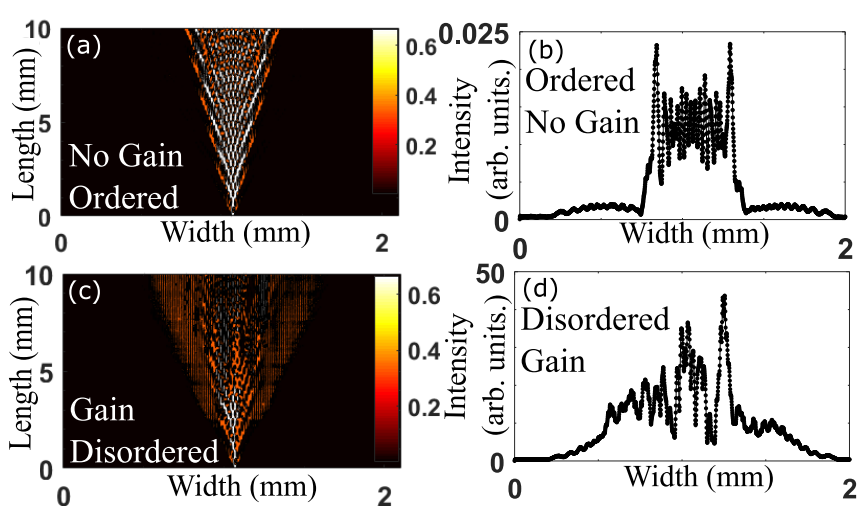

FIG. 3. Beam dynamics and Lévy-like intensity: (a) and (b) show the evolution of a light beam through a 10-mm-long ordered lattice and the corresponding output light intensity distribution. (c) and (d) show the evolution of a light beam through the same lattice in the presence of $20 \%$ refractive index disorder and a gain of 0.0001 in the bulk as well as corresponding ensemble-averaged light intensity fluctuations at the output over 50 realizations.

an equal amount of loss to all the waveguides, whereas for the other two cases the light propagation is still in the ballistic domain. Therefore it can be concluded that the introduction of gain/loss to the lattice significantly influences the dynamics of light propagation in the presence of appropriate disorder.

\section{DIFFUSIVE DYNAMICS}

To identify the supported regime of light propagation we studied the evolution of a light beam through an ordered and 20\% disordered, 10-mm-long lattice as compared in Figs. 3(a) and 3(c), respectively. It can be observed from Fig. 3(a) that the propagation of a light beam through the ordered lattice in the absence of gain is ballistic in nature as the width of the light beam increases monotonically with distance. However, when complex coupling is added in the presence of adequate disorder, transverse spreading is enhanced and thus the width of the light beam increases nonlinearly with distance, giving rise to the phenomenon of superdiffusion due to the effect of anomalous delocalization [21]. This superdiffusive nature of transport can be further confirmed by calculating the ensemble-averaged mean-squared displacement (eMSD) $\left\langle x^{2}\right\rangle=D t^{\gamma}$. Here, $D$ is the diffusion constant and $\gamma$ determines the diffusive nature of the transport. For superdiffusive transport eMSD increases faster than linearly with time $t$, which means that $\gamma$ is more than 1 . Moreover, this superdiffu- sive nature of light propagation can also be confirmed from the Lévy nature of the intensity fluctuation, as can be compared from Figs. 3(b) and 3(d). This Lévy nature of the intensity fluctuations also confirms that the random walk of photons within this active disordered lattice also follows a Lévy-like random walk similar to that in Lévy glass.

To study the origin of such intensity fluctuations as shown in Fig. 3 we have calculated the particlelike motion through this disordered lattice in the presence of various disorder amounts analogous to a center of mass (c.m.) of $\sum_{n} \frac{n\left|\psi_{n}\right|^{2}}{P}$, where $\psi_{n}$ is the beam envelope at the $n$th lattice site and $P$ is the total conserved power of the lattice [20]. Figures 4(a) and 4(b) represent the light behavior for a passive and active lattice, respectively. We can see that the distribution of random walk at the lattice end for the passive lattice is much wider in comparison to the active lattice. This arises because the disorder in the presence of gain causes the effective nonuniformity in the disorder of the system to be enhanced, which causes the onset of anomalous localization. Moreover, we have also calculated the ensemble-averaged mean-squared displacement (eMSD),

$$
\left\langle x^{2}(z)\right\rangle=\frac{1}{n_{r}} \sum_{i=1}^{n_{r}} x_{i}^{2}(z),
$$

where $x_{i}$ is the c.m. for the lattice as described above. The results are shown in Fig. 4(c). Here, we have compared the eMSD of the disordered lattices in the presence and absence of gain in reference to the eMSD of an ordered lattice. The green solid line having a constant slope depicts the eMSD of an ordered lattice. However, the deliberate presence of transverse refractive index disorder dictates the light propagation will enter the subdiffusive domain, as shown by the dotted black curve at $\gamma<1$. But when gain is introduced to make the lattice active in nature, the light experiences a superdiffusive nature of propagation, as indicated by $\gamma>1$ shown in the dashed red curve. Thus to observe these exotic phenomenon of light, introducing a little gain is just right. Moreover, the introduction of selective gain opens up another avenue of research in the field of random lasers using such lattices as a versatile platform.

\section{ROLE OF PATTERNED GAIN}

Now, to further establish the role of gain, we have observed the evolution of a light beam through active lattices of two different lengths. Two different lengths are considered because the effect of gain upon light propagating through a longer
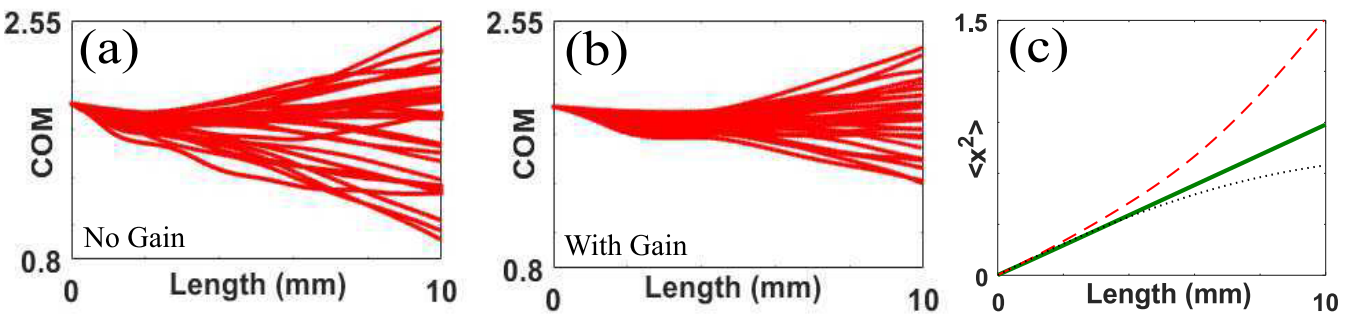

FIG. 4. (a) and (b) Random walk of photons through a 10-mm-long disordered lattice in the absence and presence of gain, respectively. (c) Superdiffusive regime: Ensemble-averaged mean-squared displacement (eMSD). 

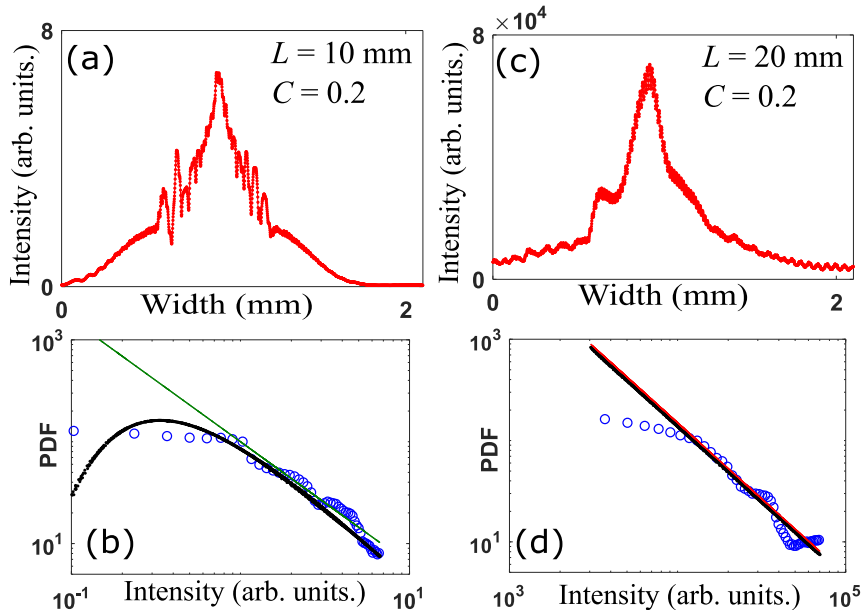

FIG. 5. Probability density function: (a) and (b) Ensembleaveraged light intensity fluctuations of 200 realizations at the end of a $20 \%$ disordered active lattice of length $10 \mathrm{~mm}$ and the corresponding PDF plot. (c) and (d) Corresponding plots for a 20-mm counterpart. In (b) and (d) the dotted black line represents the $\alpha$-stable fit, the solid lines represent the inverse-law function fit, and the blue circles denote the scattered data points for intensity.

lattice would be more. The probability density function (PDF) of the intensity fluctuations have been calculated and studied using the $\alpha$-stable law which is an econophysical function for fitting the statistical data $[22,23]$. This special function is used because intensity fluctuation involves mixed statistics and thus the method of histogram fitting fails to identify the regime of operation. The $\alpha$-stable law comprises only four parameters arranged in the following way,

$$
\ln \phi(t)=-\sigma^{\alpha}|t|^{\alpha}\left\{1-i \beta \operatorname{sgn}(t) \tan \frac{\pi \alpha}{2}\right\}+i \mu t, \quad \alpha \neq 1,
$$

$\ln \phi(t)=-\sigma|t|\left\{1-i \beta \operatorname{sgn}(t) \frac{2}{\pi}\right\}+i \mu t, \quad \alpha=1$,

where $\alpha \in(0,2]$ describes the rate at which the tails taper off of the function $\phi(t)$ which is the inverse Fourier transform of PDF. For $\alpha<2$ we observe a heavy-tailed distribution indicating Lévy behavior, whereas with $\alpha=2$ the distribution indicates Gaussian behavior. The other three parameters are the location parameter $\mu$, the skewness parameter $\beta$, and the width $\sigma$, which respectively describe the asymmetry, the mean, and the width of the distribution. The ensembleaveraged light intensity fluctuations at the output of the optical lattices as well as their analysis are depicted in Fig. 5. The comparison of the ensemble-averaged light intensity fluctuations for active lattices of 10 and $20 \mathrm{~mm}$ length having a gain value of 0.0001 are shown in Figs. 5(a) and 5(c), respectively. The corresponding PDF plots are shown in Figs. 5(b) and 5(d). Here, the black dotted line represents the $\alpha$-stable fit, the scattered data points of the ensemble-averaged light intensity are shown by blue scattered circles, and the solid lines depict the inverse power-law function fits. It can be seen that an active lattice with a longer length reveals Lévy characteristics. This can be further confirmed by fitting these PDFs against the inverse power-law function $1 / I^{\alpha}$, where $I$ is the intensity
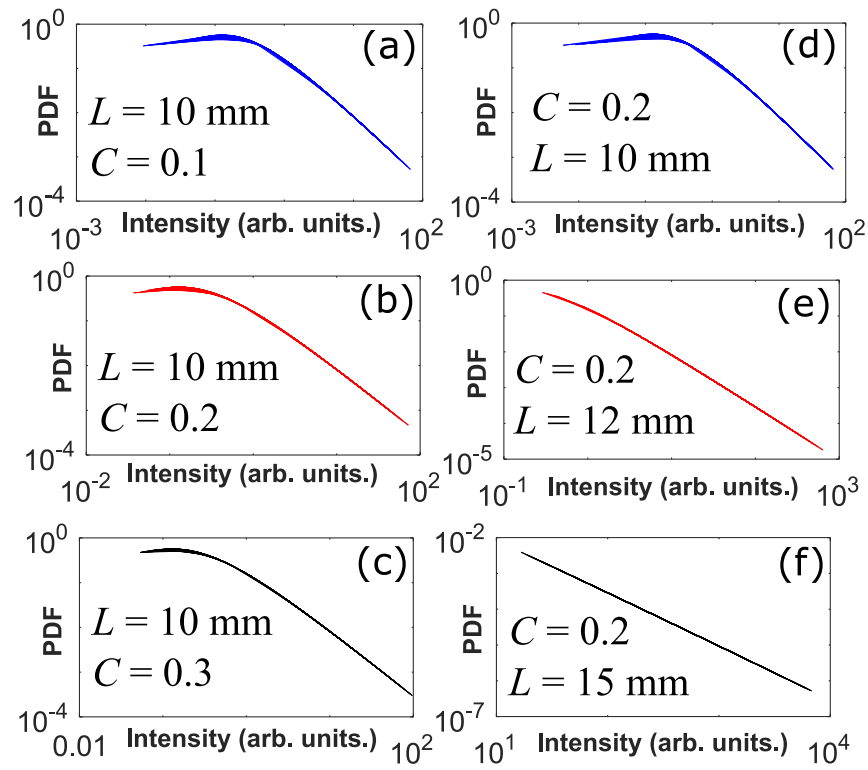

FIG. 6. Direct comparison of PDFs to establish Lévy formation: (a)-(c) compare the PDF plots for a 10-mm-long active lattice in the presence of $10 \%, 20 \%$, and $30 \%$ disorder, respectively, whereas (d)-(f) compare the PDF plots for an active lattice of lengths 10, 12, and $15 \mathrm{~mm}$, respectively, in the presence of $20 \%$ disorder.

and $\alpha$ is the slope. The PDF which fits more accurately to an inverse power-law profile is considered to exhibit a better Lévy-like intensity fluctuation. Moreover, the superdiffusive propagation regime of light is reestablished for $\alpha>1$, which is satisfied in our case by an inverse power-law fit. The active lattice having a $20 \mathrm{~mm}$ length exhibits better Lévy formation as its PDF resembles the inverse power-law profile over a larger intensity range as compared to the $10-\mathrm{mm}$ lattice. The origin of such a phenomenon owes itself to the light beam experiencing a gain medium for a longer period during propagation through this $20-\mathrm{mm}$ lattice. Thus a general query arises regarding the optimum amount of gain and disorder which should be applied to the perfectly ordered lattice in order to observe a Lévy-like intensity fluctuation and in turn explore the possibilities of a disordered lasing phenomenon. Therefore in the preceding section we have studied the interplay between the disorder and gain separately.

\section{SIGNATURE OF LÉVY FLIGHT}

A direct comparative study on the basis of the interplay between disorder and lattice length to find the optimum condition for the onset of Lévy distribution has been performed. In this section the transition from a ballistic to superdiffusive regime of light propagation will be established. It will also be reconfirmed that the role of gain is inevitable for the formation of a Lévy-like intensity fluctuation. To justify our claims we have calculated the PDFs of the output intensity fluctuations through different lattices. The PDFs shown in Fig. 6 are fitted according to the $\alpha$-stable law described earlier, however, unlike the previous plot (Fig. 5), we have not shown the data point for the intensity profile. For our analysis, a homogeneous gain of 0.0001 is introduced to the bulk of all the lattices chosen here. The PDF plots of the 
$10-\mathrm{mm}$ active lattice in the presence of $10 \%, 20 \%$, and $30 \%$ disorder are shown by Figs. 6(a)-6(c), respectively. It can be observed that the tail of the PDF is most extended for the lattice having maximum disorder. However, though we have a favorable outcome on the formation of a Lévy-like intensity pattern due to the enhancement of disorder, it demands further improvement. Thus for a better appreciation we have studied the scenario where the light beam could experience the effect of gain for a much longer period. This time we have kept the disorder fixed at $20 \%$ and the homogeneous gain is fixed at a value of 0.0001. Disordered lattices of lengths 10, 12, and $15 \mathrm{~mm}$ are studied. The lengths are chosen such that we could observe the transition from a ballistic to superdiffusive regime of light propagation. The calculated PDFs of the output intensity fluctuations through these lattices are shown in Figs. 6(d)-6(f), respectively. It can be seen that the PDF of the longest lattice has the most heavy-tailed profile and matches most accurately with the inverse power-law function. From the PDF shown in Fig. 6(d) it can be inferred that the intensity fluctuation for the $10-\mathrm{mm}$ lattice is far from the Lévy distribution and resembles more a Gaussian distribution for a lower-intensity range. However, as the lattice length is increased, we see a gradual increase in the heavy-tailed nature of the PDF. This scenario resembles the transition of a light propagation regime from ballistic to diffusive and finally to the superdiffusive regime generating a Lévy-like intensity fluctuation. These results establish the inevitable role of gain in the formation of a Lévy-like intensity fluctuation and in turn a disordered lasing effect. We may also highlight here that the superdiffusion is essentially a conservative process of spreading, while the total number of particles are conserved. This can be revealed from a direct comparison of the effect of various disorders of $10 \%, 20 \%, 30 \%$, and $40 \%$ in Figs. 6(a)-6(d), respectively, on the PDF plots (the PDF for $40 \%$ disorder has not been shown in Fig. 6). Here, we study the designed structure for different disorders by maintaining a constant amplification factor, i.e., by considering the lattice length to be fixed at $10 \mathrm{~mm}$. Hence, we have established Lévy flight in the absence of scatterers following a Lévy distribution. Additionally, a critical transition distance from a ballistic to diffusive domain is reduced and also a superdiffusive propagation distance has been revealed after a certain propagation distance due to the interplay of gain/loss and disorder. We have established these observed behaviors as stable propagation characteristics in such systems unlike any transitory behavior.

\section{CONCLUSION}

In summary, the light beam dynamics through a disordered lattice having a complex refractive index is reported. The transition of light propagation from a ballistic to diffusive domain and finally forming a Lévy pattern is demonstrated, indicating a superdiffusive regime of light propagation. Such outcomes are reported out of a 1D waveguide lattice, unlike previous claims where the distribution of scatterers follows Lévy statistics within the material. In this regime the interplay of disorder and waveguide coupling has resulted in a Lévylike distribution of the ensemble-averaged intensity profile. Therefore it can be concluded that a little gain is enough in the presence of adequate disorder in the formation of a Lévy distribution of a superdiffusive light state. We strongly believe that the findings will help in better understanding the workings of disordered lasers as open quantum systems in the presence of gain and disorder and may also find applications in image transport/projection. Moreover, deliberate and customized losses in waveguides (instead of gain in the bulk) should result in a similar light dynamics with a characteristic Lévy tail. Hence, disorder, the amount of gain/loss, and the lattice aspect ratio are the key parameters. The results are reported in a diffusive regime away from the localized and ballistic regime.

\section{ACKNOWLEDGMENTS}

S.B. acknowledges the financial support from Ministry of Human Resource Development (India). S.G. acknowledges the financial support by the Science and Engineering Research Board (SERB), India under Early Career Research Grant (ECR/2017/000491).
[1] T. Schwartz, G. Bartal, S. Fishman, and M. Segev, Nature (London) 446, 52 (2007).

[2] Y. Lahini, A. Avidan, F. Pozzi, M. Sorel, R. Morandotti, D. N. Christodoulides, and Y. Silberberg, Phys. Rev. Lett. 100, 013906 (2008).

[3] E. Altshuler and E. Shimshoni, Phys. Rev. B 45, 2712 (1992).

[4] P. Barthelemy, J. Bertolotti, and D. S. Wiersma, Nature (London) 453, 495 (2008).

[5] M. Golshani, S. Weimann, Kh. Jafari, M. K. Nezhad, A. Langari, A. R. Bahrampour, T. Eichelkraut, S. M. Mahdavi, and A. Szameit, Phys. Rev. Lett. 113, 123903 (2014).

[6] A. Amir, Y. Lahini, and H. B. Perets, Phys. Rev. E 79, 050105(R) (2009).

[7] C. Mejía-Cortés and M. I. Molina, Phys. Rev. A 91, 033815 (2015).
[8] A. K. Harter, F. A. Onanga, and Y. N. Joglekar, Sci. Rep. 8, 44 (2018).

[9] S. Longhi, D. Gatti, and G. D. Valle, Sci. Rep. 5, 13376 (2015).

[10] D. M. Jović, C. Denz, and M. R. Belić, Opt. Lett. 37, 4455 (2012).

[11] Y. V. Kartashov, V. A. Vysloukh, V. V. Konotop, and L. Torner, Phys. Rev. A 93, 013841 (2016).

[12] A. Bahrampour, E. Shojaie, and M. Sani, J. Opt. Soc. Am. B 31, 1308 (2014).

[13] Y. Wu, Y. Ren, A. Chen, Z. Chen, Y. Liang, J. Li, G. Lou, H. Zhu, X. Gui, S. Wang, and Z. Tang, Nanoscale 9, 6959 (2017).

[14] A. S. L. Gomes, E. P. Raposo, A. L. Moura, S. I. Fewo, P. I. R. Pincheira, V. Jerez, L. J. Q. Maia, and C. B. de Araújo, Sci. Rep. 6, 27987 (2016).

[15] H. Cao, Y. G. Zhao, S. T. Ho, E. W. Seelig, Q. H. Wang, and R. P. H. Chang, Phys. Rev. Lett. 82, 2278 (1999). 
[16] S.-W. Chang, W.-C. Liao, Y.-M. Liao, H.-I Lin, H.-Y. Lin, W.-J. Lin, S.-Y. Lin, P. Perumal, G. Haider, C.-T. Tai, K.-C. Shen, C.-H. Chang, Y.-F. Huang, T.-Y. Lin, and Y.-F. Chen, Sci. Rep. 8, 2720 (2018).

[17] S. Lepri, S. Cavalieri, G.-L. Oppo, and D. S. Wiersma, Phys. Rev. A 75, 063820 (2007).

[18] E. Ignesti, F. Tommasi, L. Fini, S. Lepri, V. Radhalakshmi, D. Wiersma, and S. Cavalieri, Phys. Rev. A 88, 033820 (2013)
[19] S. Ghosh, N. D. Psaila, R. R. Thomson, B. P. Pal, R. K. Varshney, and A. K. Kar, Appl. Phys. Lett. 100, 101102 (2012).

[20] S. Flach and A. V. Gorbach, Phys. Rep. 467, 1 (2008).

[21] S. Yu, X. Piao, and N. Park, ACS Photonics 5, 1499 (2018).

[22] R. Uppu, A. K. Tiwari, and S. Mujumdar, Opt. Lett. 37, 662 (2012).

[23] R. Uppu and S. Mujumdar, Phys. Rev. A 90, 025801 (2014). 